\title{
CASE REPORT \\ Microvascular free flap in a pregnant patient after resection of a high-grade neuroendocrine carcinoma
}

\author{
Tatiana Luna-Pisciotti, Mariana Izquierdo, María P. Echeverri, Alejandra Sanín and \\ Luis Nieto* \\ Hospital Universitario San Ignacio, Faculty of Medicine, Pontifical Xavierian University, Bogotá, Colombia
}

Abstract

Free flap reconstruction is seldom performed during pregnancy. Not only does the prolonged operative time pose a risk for the mother and the fetus, but also the hypercoagulable state of pregnancy predisposes the mother to a greater risk of complications in the transplanted tissue. We present a case of a 29-year-old patient in week 27 of gestation with a rapidly progressive neuroendocrine tumor in the left nasal fossa with involvement of the nasal sinus, pterygopalatine fossa, dura, and left orbit, associated with neurological symptoms and recurrent epistaxis. The aggressive and rapidly progressive character of the tumor made surgical excision by a multidisciplinary team as the first option. We performed immediate reconstruction with an anterolateral thigh free flap. Free tissue transfer was performed successfully, with satisfactory results on the mother and later delivery without complications.

Keywords: microsurgical free flaps; free tissue flap; gestation; pregnancies; neuroendocrine tumor; head and neck cancer

Received: I 8 June 2021; Revised: 29 August 2021; Accepted: 7 September 2021; Published: I8 October 202 I

$\mathrm{M}$ icrovascular tissue transfer during pregnancy was first reported by Haws et al. in 1996 (1). The authors present a case of successful arm reimplantation on a 26-year-old patient, with later delivery without complications. Lloyd et al. in 2003 described the case of a 36-year-old patient in her third trimester of pregnancy with a grade III tongue carcinoma treated with excision and reconstruction with a radial forearm free flap; this being the first reported case of a successful microvascular transfer in oral cancer during pregnancy (2). To date, only few cases of microvascular flaps during pregnancy have been reported, most of them associated with reconstruction following the resection of head and neck tumors.

Microvascular surgery is often performed in a programmed setting. Best results are obtained with appropriate planning, especially in patients with an optimal health status. During pregnancy, prolonged surgery stress and anesthetic agents might lead to fetal and maternal complications such as congenital malformations and preterm delivery. Accordingly, pregnancy has been considered a relative contraindication for microvascular tissue transfer (3). When absolutely necessary, as in the case of reconstruction after resection of aggressive head and neck tumors, the second trimester of gestation is considered the safest time for anesthetic procedures, when organogenesis is complete, and the risk of preterm labor is low (4). A case of successful microvascular transfer following resection of a high-grade neuroendocrine head and neck tumor during pregnancy is presented.

\section{Case report}

A 29 -year-old patient in week 26 of gestation consulted for left hemicraneal headache, retroocular pain, nasal congestion, and moderate recurrent epistaxis. On examination, a mass in the left nasal fossa in contact with nasal conchae, left eye proptosis with limitation for abduction, and diplopia with supraduction and abduction were evident (Fig. 1). A biopsy was performed with pathology compatible with high-grade large cell neuroendocrine carcinoma. Orbit and cranial Magnetic Resonance Imaging (MRI) revealed an extension of the lesion to the left orbit, maxillary sinus, and left pterygopalatine fossa, with localized dural enhancement (Fig. 2). No evidence of metastatic involvement was found in further images, making surgical treatment the first option.

On week 27 of gestation, the procedure was performed by a multidisciplinary surgical team of otorhinolaryngologists, neurosurgeons, ophthalmologists, maxillofacial surgeons, head and neck surgeons, and plastic surgeons, under general anesthesia. Rapid sequence induction was achieved with fentanyl, lidocaine, propofol, 
succinylcholine, and precurarization with rocuronium. Invasive central venous and arterial pressure monitoring were employed. Anesthesia was maintained with remifentanil and sevoflurane, and neuromuscular relaxation was obtained with pancuronium. Surgery consisted in the exenteration of the left orbit, preserving $30 \%$ of the left upper and lower eyelids, superstructure maxillectomy, frontal sinusotomy, endoscopic anterior and posterior ethmoidectomy, antrostomy, and left sphenoidotomy, with a resulting orbito-maxillary defect Cordeiro type IIIb (5) (Fig. 3). We designed an anterolateral tight fasciocutaneous free flap to reconstruct the defect. The descending branch of the lateral circumflex femoral artery and its vena comitans were anastomosed to the left superficial temporal vessels in end-to-end fashion. Heparin-saline solution (5,000 units) was used to irrigate the anastomotic vessels. We achieved obliteration of dead spaces in the orbit and maxillary sinus, and adequate reconstruction of the palate. No systemic heparin was administered during the procedure, and thromboembolic prophylaxis was established with intermittent pneumatic pressure stockings. Finally, the airway was secured with a tracheostomy.

The total operative time was $14 \mathrm{~h}$, during which permanent cardiotocographic monitoring of fetal wellbeing was

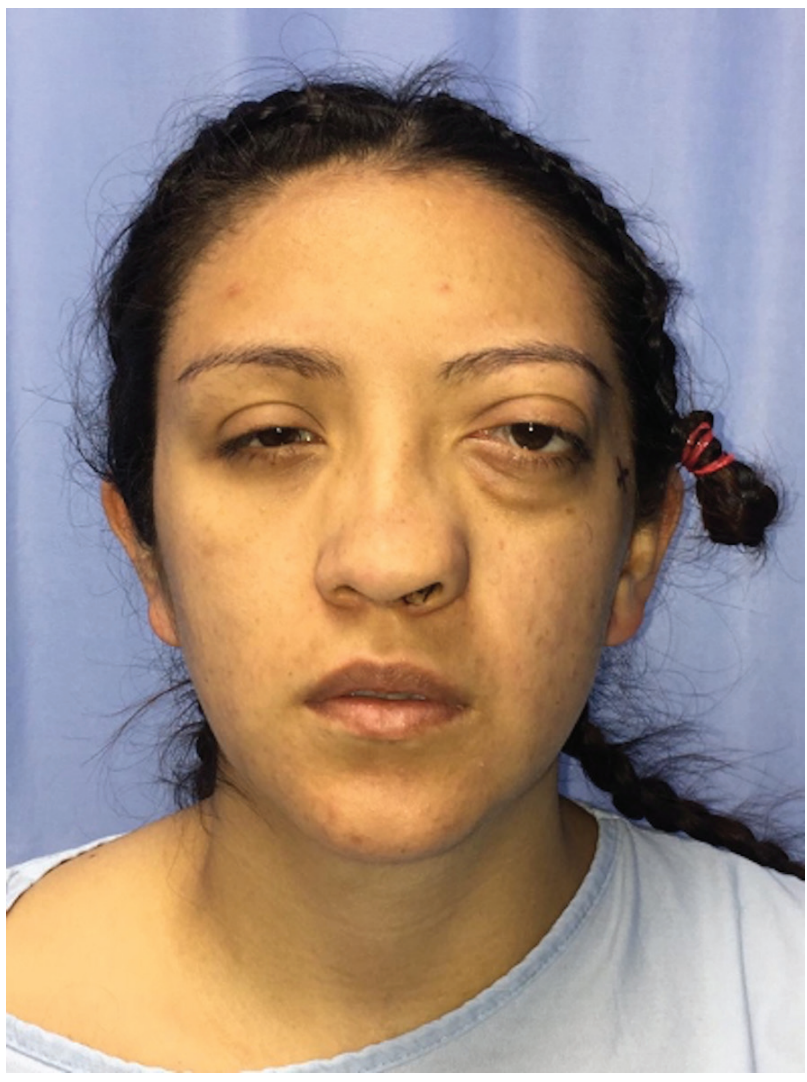

Fig. 1. Oculo-facial deformity due to tumor. established by the obstetric team. There were no inconveniences on the mother or the fetus during the procedure. Thromboprophylaxis was achieved with Low molecular weight heparin (LMWH) and compression stockings during hospital stay. The postoperative course was uneventful, and we observed successful flap survival without complications.

On week 34 of gestation, a programmed cesarean delivery was performed. A healthy preterm baby girl was born weighing 1,600 g. Histopathology revealed highgrade neuroendocrine large cell carcinoma with positive margins. The patient received adjuvant radiotherapy with a satisfactory evolution and complete flap survival at the time of writing this manuscript (10 months after surgery) (Fig. 4).

\section{Discussion}

Head and neck neuroendocrine tumors constitute a group of rare and morphologically heterogeneous tumors. Non-squamous carcinomas represent $1 \%$ of the malignant tumors in head and neck, of which $37 \%$ are neuroendocrine carcinomas (6). In the USA, 853 cases of poorly differentiated small cell neuroendocrine carcinomas have been identified. This type of tumor is characterized by an aggressive progression with initial non-specific symptoms, rapid systemic dissemination, and short survival rates (7).

Head and neck tumors are rare in pregnant patients. When they do occur, the therapeutic possibilities are limited by the presence of the fetus and the physiological

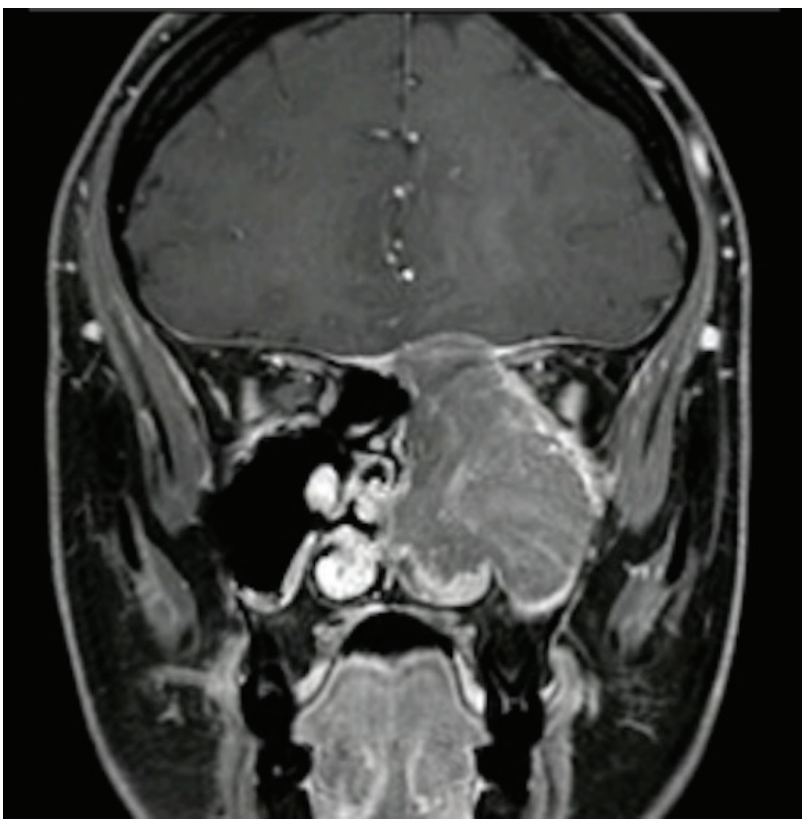

Fig. 2. MRI presenting the extension of the tumor in the left orbit, maxillary sinus, left pterygopalatine fossa, and localized dural enhancement. 
changes that arise during pregnancy. Surgical excision with immediate reconstruction is often one of the first therapeutic alternatives, given that radiotherapy carries a

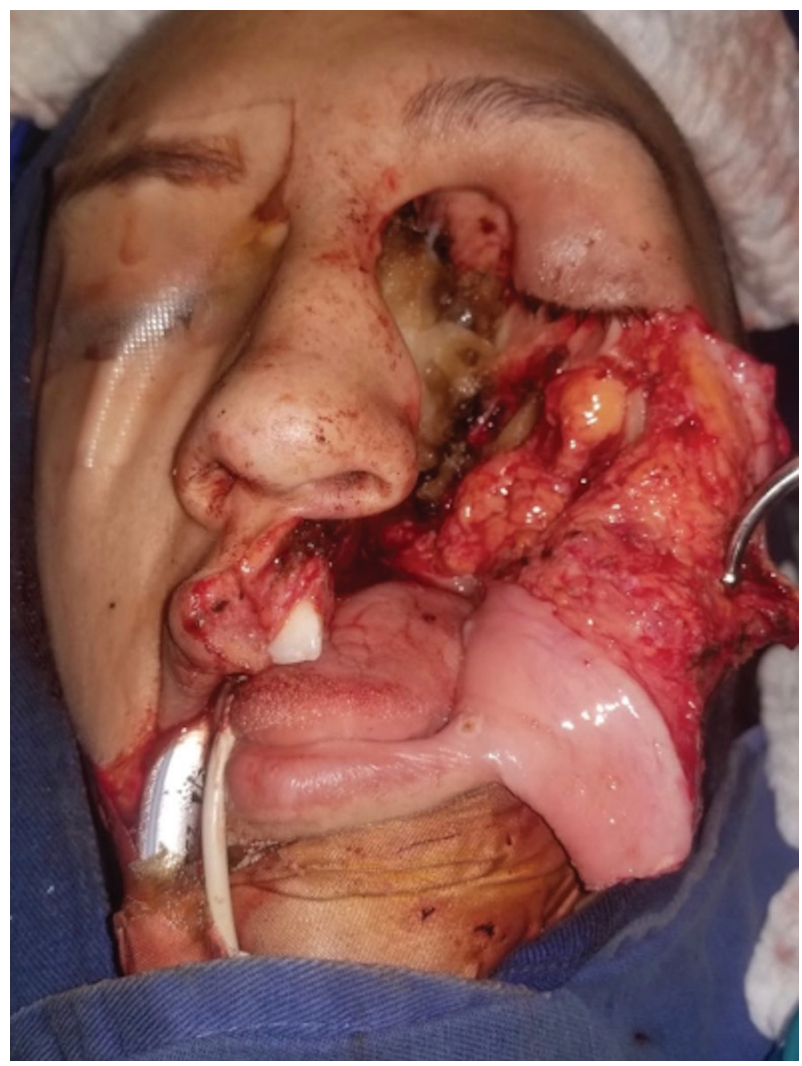

Fig. 3. Orbito-maxillary defect posterior to tumor resection. high risk for cancer during childhood in the unborn child and new primary cancer for the mother (8). Physiological changes during pregnancy hinder the performance of a microvascular flap for defect reconstruction. Circulating fibrin and other coagulation factors rise, platelet exchange is accelerated, and fibrinolytic activity is reduced. These processes contribute to a hypercoagulable state, increasing the risk of anastomosis thrombosis and thromboembolic complications (9).

In addition to the technical difficulties presented to the surgical team during pregnancy, management of the anesthetic requires careful preparation for prolonged surgery. The use of pregnancy-safe anesthetic agents and continuous monitoring of the mother and the fetus are required. Furthermore, blood loss and hypotension in the mother should be managed on a higher threshold, since any level of systemic hypoperfusion would compromise fetal wellbeing (10).

\section{Conclusion}

Reflecting what is reported in the literature, we presented the case of a patient in her second trimester of gestation, in whom a successful reconstruction with a microvascular flap was performed after the resection of a high-grade neuroendocrine tumor of the left paranasal sinus. Free tissue transfer during pregnancy can be a challenge for the microsurgeon. However, with adequate planning and perioperative care, and warranting optimal thromboprophylaxis, it can be performed successfully. Microvascular surgery should not be disregarded as a reconstructive alternative in this group of patients.

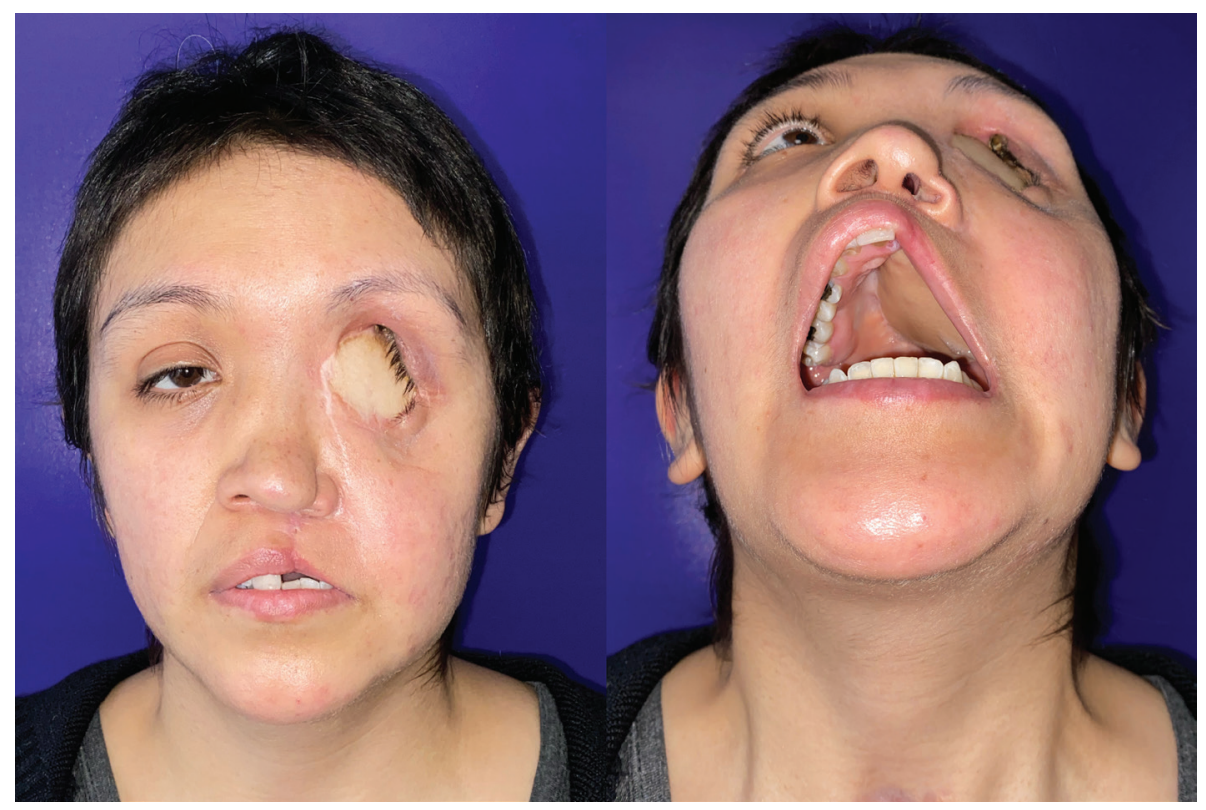

Fig. 4. Postoperative results 10 months after surgery. 


\section{Acknowledgments}

No acknowledgements to present.

\section{Conflicts of interests and funding}

The authors pose no conflicts of interest to declare. The authors received no financial support for the research, authorship, and/or publication of this article.

\section{Ethics statement}

A written informed consent was obtained from the subjects who included in this report.

\section{Authors' contributions}

The authors declare having participated in the elaboration of this manuscript. Luis E. Nieto was involved in the conception and design of the manuscript, and in the formulation of the discussion and conclusions from the plastic surgery point of view. Tatiana M. Luna and Mariana Izquierdo were involved in the collection of images and data, and the writing and editing of the manuscript. Maria P. Echeverri and Alejandra Sanin were involved in the case analysis and the development of the discussion and conclusions from the anesthesiology point of view. All the authors contributed to the revision of the manuscript, which has been approved by each one of them for its submission and publication.

This manuscript was performed under Pontifical Xaverian University IRB approval.

\section{References}

1. Haws MJ, Erdmann D, Brown RE. Above-elbow arm replantation during pregnancy. Ann Plast Surg 1996; 36: 93-6. doi: 10.1097/00000637-199601000-00019

2. Lloyd CJ, Paley MD, Penfold CN, Varadarajan V, Tehan B, Gollins SW. Microvascular free tissue transfer in the management of squamous cell carcinoma of the tongue during pregnancy. Br J Oral Maxillofac Surg 2003; 41(2): 109-11. doi: 10.1016/ S0266-4356(03)00003-2

3. Al Qattan MM, Bowen V. Effect of pre-existing health conditions on the results of reconstructive microsurgery. Microsurgery 1993; 14: 152-7. doi: 10.1002/micr.1920140304

4. Meger GR, Majewski WT, Lyle WG. Free tissue transfer in pregnancy: guidelines for perioperative management. Microsurgery 2001; 21(5): 202-7. doi: 10.1002/micr.1039. PMID: 11494393

5. Cordeiro PG, Chen CM. A 15-year review of midface reconstruction after total and subtotal maxillectomy: part I. Algorithm and outcomes. Plast Reconstr Surg 2012; 129(1): 124-36. doi: 10.1097/PRS.0b013e318221dca4

6. Torabi SJ, Cheraghlou S, Kasle DA, Savoca EL, Judson BL. Nonsquamous cell laryngeal cancers: incidence, demographics, care patterns, and effect of surgery. Laryngoscope 2019; 129(11): 2496-505. doi: 10.1002/lary. 27785

7. Van der Laan TP, Plaat BE, van der Laan BF, Halmos GB. Clinical recommendations on the treatment of neuroendocrine carcinoma of the larynx: a meta-analysis of 436 reported cases. Head Neck 2015; 37: 707-15. doi: 10.1002/hed.23666

8. Antonelli NM, Dotters DJ, Katz VL, Kuller JA. Cancer in pregnancy: a review of the literature. Part I. Obstet Gynecol Surv 1996; 51: 125-34. doi: 10.1097/00006254-199602000-00022

9. Miyazaki Y, Fukuda K, Fujita K, Nishimoto S, Terada T, Wada $\mathrm{R}$, et al. Free flap transfer reconstruction in managing tongue carcinoma during pregnancy. J Surg Case Rep 2017; 2017(8): 1-4. doi: $10.1093 /$ jscr/rjx 164

10. Po G, Olivieri C, Rose CH, Saccone G, McCurdy R, Berghella V. Intraoperative fetal heart monitoring for non-obstetric surgery: A systematic review. Eur J Obstet Gynecol Reprod Biol 2019; 238: 12-19. doi: 10.1016/j.ejogrb.2019.04.033

\section{*Luis Nieto}

Pontifical Xavierian University

Cra. 7 \#No. 40 - 62

Bogotá, DC

Colombia

Email: luis-nieto@javeriana.edu.co 